\title{
Effective forming strategy for double-sided incremental forming considering in-plane curvature and tool direction
} \author{
aKornel F. Ehmann, aJian Cao (1)* \\ ${ }^{a}$ Department of Mechanical Engineering, Northwestern University, Evanston, IL, USA

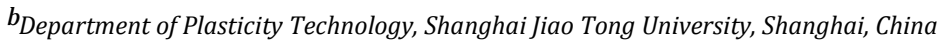 \\ \#Equal contribution
}

aNewell Moser\#, aZixuan Zhang\#, aHuaqing Ren, a,bHuan Zhang, aYi Shi, aEbot Ndip-Agbor, bBin Lu, bJun Chen,

The success of a toolpath in Double-Sided Incremental Forming (DSIF) is strongly related to the specified tool gap. It is hypothesized in this work that maintained contact between tools and the sheet can improve the distribution of sheet thickness and hence, improve material formability and prevent premature fracture. Simulation and experimental studies reveal that thickness prediction models solely dependent on the local wall angle are inadequate for general part geometries. A 'Shamrock' geometry is proposed leading to the development of a novel improved thickness correction model that incorporates wall angle, in-plane curvature, and tool direction.

Incremental sheet forming, Process control, Toolpath

\section{Introduction}

Double-Sided Incremental Forming (DSIF) is a flexible, die-less manufacturing process that utilizes generic tooling to locally deform sheet metal into freeform geometries. By using two tools, one above and one below the clamped sheet, highly-customized parts can be formed using toolpaths generated directly from a CAD part (Fig. 1). Although Single-Point Incremental Forming (SPIF) exhibits excellent material formability [1] and the potential for closed-loop accuracy feedback [2], DSIF provides increased control during material deformation [3] resulting in enhanced geometric accuracy [4] and also further increasing formability [5].
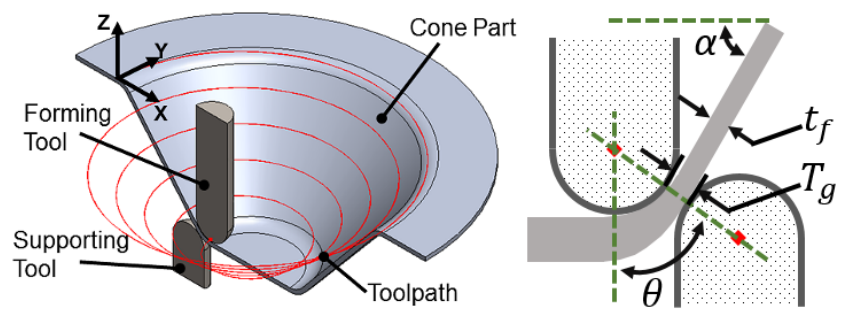

Fig. 1. (left) Cross-section of DSIF, (right) tool position parameters.

The relative tool positioning of the two tools is given by Fig. 1, where $\alpha$ is the wall angle, $\theta$ is the angle between the tools with respect to the Z-axis, $\mathrm{t}_{\mathrm{f}}$ is the deformed thickness, and $\mathrm{T}_{\mathrm{g}}$ is the tool gap. While closed-loop control of tool positioning based on forming forces in situ of DSIF has been investigated by Meier et al. [6], the use of displacement control is still an attractive option for general parts in industry because of the simplicity in machine and controller design, as well as fast response time and robustness to variations in material properties. However, estimates of sheet thinning and machine compliance must be calculated prior to physical forming in order to prevent the supporting tool from losing contact with, or over-squeezing, the sheet metal.

The study presented in this paper was motivated by an observation found in experiments regarding the importance of maintaining tool contact. Figure 2 illustrates a DSIF-formed circular funnel. Interestingly, fracture occurred within the section of sheet which lost contact with the supporting tool, while the opposing region maintained contact with both tools and evidently did not experience fracture. Similar discoveries were observed related to the location of fracture initiation for other parts formed by DSIF, including a truncated pyramid. In the event that the supporting tool loses contact with the sheet, the DSIF process inherently degenerates into SPIF, thus eliminating the throughthe-thickness pressure provided by the supporting tool which helps to delay fracture [7]. To maximize formability in DSIF, our hypothesis is that one must maintain both tool contacts during the entire DSIF process.

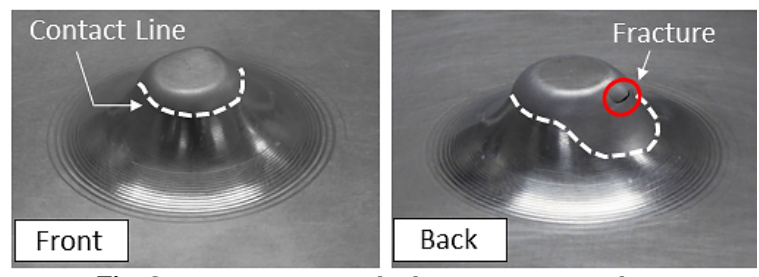

Fig. 2. Fracture initiated where contact was lost.

Factors that can contribute to the loss of tool contact with the sheet typically involve either inaccurate thickness prediction, tool misalignment, or unaccounted for machine compliance. In Fig. 2, the part is axisymmetric and lost contact likely due to a tool misalignment. However, inaccurate thickness prediction is the most common culprit. In incremental sheet forming, the part's thickness is often predicted by the Sine law, $t_{f}=t_{0} \sin \left(90^{\circ}-\alpha\right)$ where $t_{0}$ is the initial thickness. However, the Sine Law is 
dependent on just the wall angle and thus is not well-suited for general features.

Our investigation of the relation between wall angle and loss of contact initiated with the truncated pyramid geometry (Fig. 3). This part geometry contains four sides with different, constant wall angles, though, contact was still lost in some regions. In particular, it was observed that contact was lost at the corners between the four sides implying that part curvature likely plays a role in the loss of contact between the sheet and the tools.

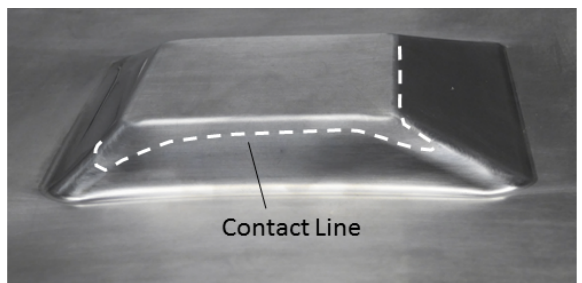

Fig. 3. Asymmetric contact loss on truncated pyramid part.

\section{Analysis of the in-plane curvature effect on thickness}

In this work, we will prove the need to use in-plane curvature when compensating for machine compliance and sheet thinning for DSIF toolpaths. Additionally, a novel correction model is presented and demonstrated on a part geometry, named the 'Shamrock' part, specifically designed to decouple wall angle from in-plane curvature as shown in Fig. 4. The Shamrock part has a combination of both concave and convex in-plane curvatures, and its wall angle is kept constant around the contour regardless of the in-plane curvature. The existence of four repeating regions is to examine tool alignment or material anisotropy.
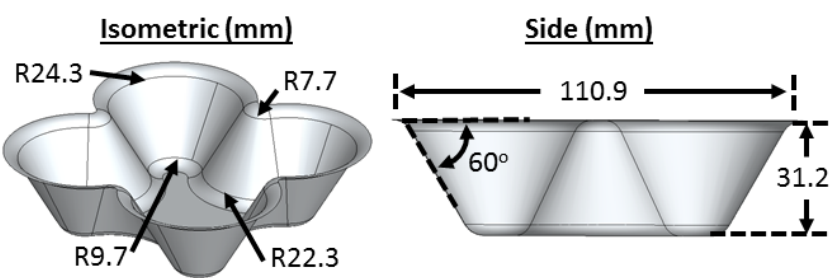

Fig. 4. 'Shamrock' part with constant wall angle.

\subsection{Curvature definition and its relation with tool movement}

Based on the local in-plane contour of the geometry, different regions of the part can be defined as either 'inward' or 'outward' and either 'departing' or 'approaching'. To illustrate these concepts, the definitions are marked in Fig. 5 along with an inplane cross-section contour of the Shamrock design. Note that the motion direction of both tools is illustrated as well, in this case, they are moving in the counter-clockwise direction.

If the center of curvature lies within the interior of the contour, then the region is termed 'inward'. Contrarily, if the center of curvature lies outside the contour, then the local point is termed 'outward'. The 'approaching' and 'departing' regions are defined using $\beta$, i.e., the angle between the contour's tangent vector and the vector connecting the centroid to the local point. If the angle $\beta>90^{\circ}$, then the local region is defined as a 'departing,' whereas if $\beta<90^{\circ}$, it is defined as an 'approaching' region. Notice that the length of the vector between the local point and the centroid increases within the 'departing' region, and vice-versa for the 'approaching' region.

According to the above definitions, one leaf or corner of the Shamrock part can be divided into four different regions as shown in Fig. 5: 'outward/departing' (green), 'inward/departing' (red), 'inward/approaching' (blue) and 'outward/approaching' (magenta).

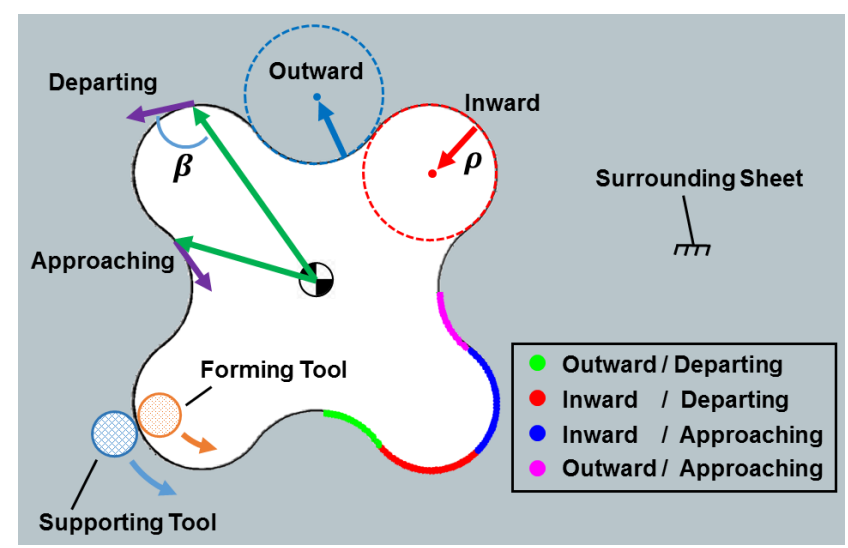

Fig. 5. One corner, or leaf, of the Shamrock part has been separated into four categories based on curvature and tool motion.

Figure 6 shows the locations of lost-contact areas in two identical parts formed using the same toolpath parameters except for the direction of tool motion. Details of the experimental parameters and conditions are given in Section 3. Two observations can be immediately made: 1) the loss of contact locations are consistent for a given part in that the region favors a particular side of the leaf; and 2) there appears to be a link between tool motion and loss of contact. The first observation eliminates tool alignment, tool asymmetry, or machine error as the causes of contact loss in our experiments. Related to the second observation, we can observe that the 'inward/ approaching' region (blue region) in both clockwise and counter-clockwise cases lost contact, while the contact is usually maintained well in the 'outward' regions (green and magenta).
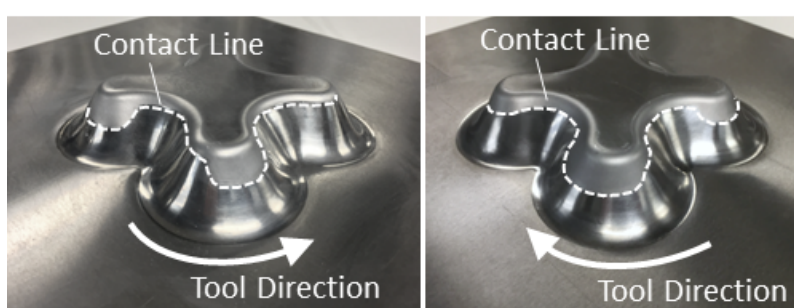

Fig. 6. Asymmetric loss of tool contact around rounded corners.

\subsection{Investigation using finite element analysis}

Simulation studies using LS-DYNA's finite element analysis (FEA) software package were performed to verify and explain the loss of contact observations found in the aforementioned Shamrock experiments. Aluminum 5754-0 (1 mm thick) was solely considered for all experiments and simulations to be discussed. As described by Moser et al. [8], an isotropic elastoplastic model ( $\mathrm{J}_{2}$-plasticity) can be utilized for this material and still capture the prevalent mechanics that occur during the DSIF process. The sheet blank ( 150 by $150 \mathrm{~mm}$ ) was modelled using a uniform grid of $0.5 \mathrm{~mm}$ shell elements, namely LS-DYNA's shell element ID 26. This particular shell element is fully integrated and has a thickness stretch in order to adequately capture through-the-thickness effects. The tools were modelled with rigid elements and were sped up to $750 \mathrm{~mm} / \mathrm{sec}$. Explicit time integration was chosen with mass scaling used to raise the stable time step to $1\left(10^{-5}\right)$ sec. Using 16 CPUs, approximately 1 week was required to complete the simulation.

In the simulation, the supporting tool lost contact with the part in an asymmetric fashion around the Shamrock's corners (Fig. 7). The Sine law was used to define the tool gap for the simulation's toolpath, which is later referred to as the 'Baseline' toolpath. Recall that the material was modelled as isotropic and the tools 
were treated as rigid, and yet, the locations where the tools lost contact were not along the middle of the Shamrock's corners, but rather, within the 'inward/ approaching' region.

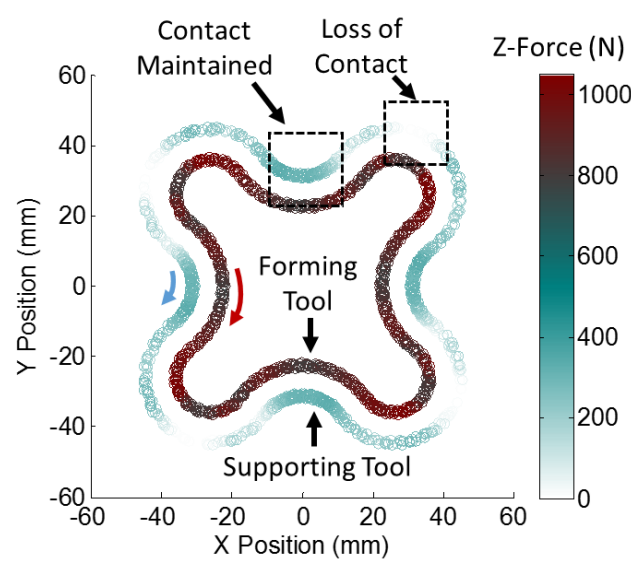

Fig. 7. Simulation Z-forces of the tools as a function of position.

Referring to the thickness distribution of the part in Fig. 8, it is clear that the sheet experienced significant thinning near the regions where the supporting tool lost contact. The illustrated thickness distribution verifies the assumption in DSIF that the induced through-the-thickness pressure provided by the supporting tool has a pivotal role in preventing premature sheet thinning. Therefore, it is imperative that the supporting tool maintains contact with the part during the forming process so as to prevent fracture.

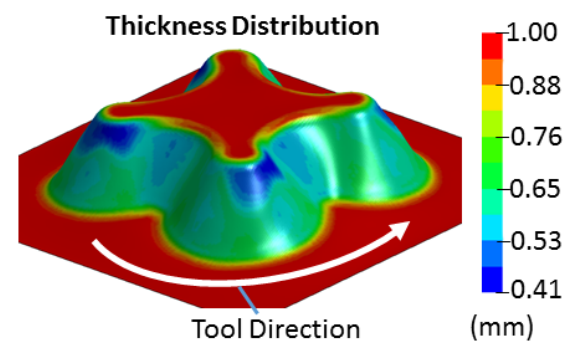

Fig. 8. Thickness distribution from the simulation of the Shamrock part with a constant $60^{\circ}$ wall angle.

Furthermore, the Shamrock part was designed with a constant wall angle of $60^{\circ}$, which implies a final thickness of $0.5 \mathrm{~mm}$ when using the conventional Sine law. However, the thickness distribution along the wall of the simulated Shamrock part is not uniform nor is approximately $0.5 \mathrm{~mm}$. This further supports the need to incorporate additional geometric features such as inplane curvature and tool direction when predicting thickness in DSIF.

\subsection{Proposed thickness prediction model that incorporates the in- plane curvature and tool movement}

In a typical DSIF toolpath, the position of the supporting tool relative to the forming tool is along the normal direction of the desired geometry, as shown in Fig. 1. The distance between the two tools is determined by the local wall angle as given by the Sine law. However, as illustrated in the previous sections, wall angle alone is not accurate in capturing the thickness change as deformation in a generic part does not follow pure shear deformation, which is assumed in the derivation of the Sine law [9].

To maintain the contact throughout the DSIF process, we propose that the position of the supporting tool is calculated using the in-plane curvature and the direction of tool motion. In the region where the part has the tendency of losing tool contact, the tool gap, $\mathrm{T}_{\mathrm{g}}$, between the two tools should be decreased. Accordingly, the corrected $\mathrm{T}_{\mathrm{g}}$ can be expressed as:

$$
T_{g}=t_{0}[1-f(h, \rho, \beta) \cdot(1-\cos \alpha)]
$$

in which $f$ is a correction function based on the feature height (h), local curvature $(\rho)$, and angle $\beta$ related to tool motion direction as defined in Figure 5. In general, $\mathrm{f}$ can be described as:

$$
f=1+a_{1}(h)\left[a_{2}(\rho)+a_{3}(\beta)\right]
$$

In Eq. 2, $\mathrm{a}_{1}$ is a function to account for machine compliance and feature springback based on feature height, while $\mathrm{a}_{2}$ and $\mathrm{a}_{3}$ represent the effects due to local curvature and $\beta$, respectively. By separating these effects, different regions regarding 'outward/inward' and 'departing/approaching' can be distinguished and thus assigned unique tool gap values. Note the chosen form of $\mathrm{f}$ in that if $\mathrm{f}=1$, then Eq. 1 will degenerate to the conventional Sine law function.

\section{Experimental validation}

\subsection{DSIF forming process parameters and equipment}

A custom-built DSIF machine at Northwestern University [8] was utilized for this study. The sheet metal used was $1 \mathrm{~mm}$ aluminum alloy AA5754-0. To ensure that the tips of the tools were spherical, a standard ball bearing with diameter of $9.5 \mathrm{~mm}$ was assembled into each tool holder. Multi-axis piezoelectric load cells were attached to the base of both tools to record the forming forces in the three orthogonal $\mathrm{X}, \mathrm{Y}$, and $\mathrm{Z}$ axes. The tools were moved along spiral toolpaths with an incremental depth, or a pitch of $0.2 \mathrm{~mm}$, and the tool speed was set to be $6 \mathrm{~mm} / \mathrm{s}$. Teflon grease was applied to the sheet metal acting as the lubricant.

While the forming tool moves along the pre-defined toolpath based on part geometry, the trajectory of the supporting tool is defined by the relative position as proposed in Section 2.3. In the Baseline study, $f=1$ from Eq. 2, i.e., the Sine law is used. In the Compensated study, the coefficients in Eq. 2 are set to $a_{1}=h / 25$, $a_{2}=0.75[1+\operatorname{sign}(\rho)]$, and $a_{3}=0.25[1+\operatorname{sign}(\cos \beta)]$, from observations. The resulting compensation for the supporting tool position is shown in Figure 9. As desired, a smaller tool gap, or larger value of $\mathrm{f}$, is assigned to areas where the supporting tool is expected to lose contact.

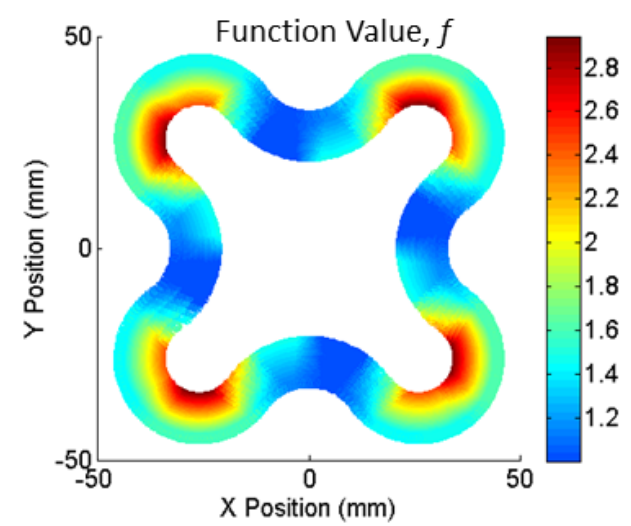

Fig. 9. Correction value, f, from Eq. 2 is plotted against position.

The Baseline and Compensated Shamrock parts are compared against each other in Fig. 10. As expected, the Compensated part exhibits nearly no regions where contact was lost. Only within the inward/approaching/ region did the correction values appear to be slightly too small and some loss of contact was observed. 
Shamrock Parts with $60^{\circ}$ Wall Angle
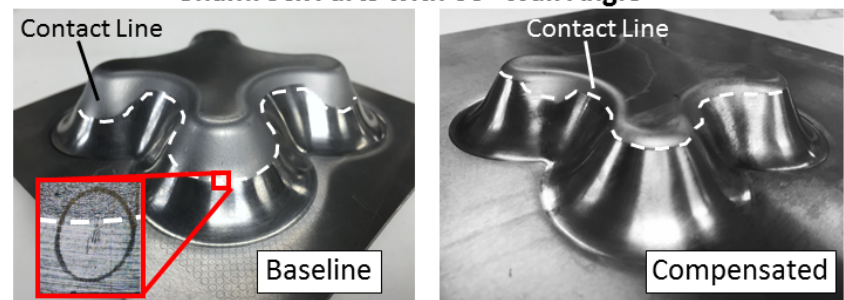

Fig. 10. Compensated toolpath maintains better contact. Strains were measured from a laser marked circle grid.

\section{Data analysis of Shamrock parts}

\subsection{Strain measurements using laser textured circular grids}

Laser marking was used to produce a reliable circle grid pattern on the sheet which was not prone to rubbing off during the DSIF process. An array of circles were marked onto the metal (Fig. 10) by a laser system at Northwestern [8] consisting of an 8 ps, pulsed Nd-YV04 laser with a $532 \mathrm{~nm}$ wavelength. Parts were formed with and without the circle grids to confirm that the resultant geometry and formability were not altered due to the gridding process.

The major and minor principal strains were generally in the meridional direction towards the centroid and the toolpath direction, respectively. Most material points approximately deformed in plane strain, though a denser grid is required in future studies for quantitative conclusions. Referring to Fig. 10, there was, however, some amount of twisting induced by shear along the toolpath direction. This twisting phenomenon is likely the reason why the direction of tool motion directly influences the region where contact is eventually lost with the supporting tool.

\subsection{Thickness distribution}

Both the internal and the external surfaces of the formed parts were measured using a Romer Absolute Arm with an integrated laser scanner. The resultant point clouds were then used to fit NURBS surfaces in Geomagic ${ }^{\circledR}$ commercial CAD software. With the parts digitized, measurements such as curvature or thickness could be readily made. A calibration plate with known thickness was used to determine the standard error for this thickness measurement, which was found to be less than $\pm 0.05 \mathrm{~mm}$. Figure 11 illustrates the 3D thickness distribution of the formed parts.

The experimental thickness distribution of the Baseline Shamrock part is in fair agreement with the values extracted from the simulation, thus verifying the trends in the preliminary simulation results. In particular, the localized necking that occurs at the corners where the supporting tool loses contact is well established. Furthermore, the thickness along the corner's wall of the Compensated Shamrock part tends to thin at a slower rate than that of the Baseline part, implying that the thickness distribution is more uniform (i.e., localized necking is less severe) when contact is maintained.

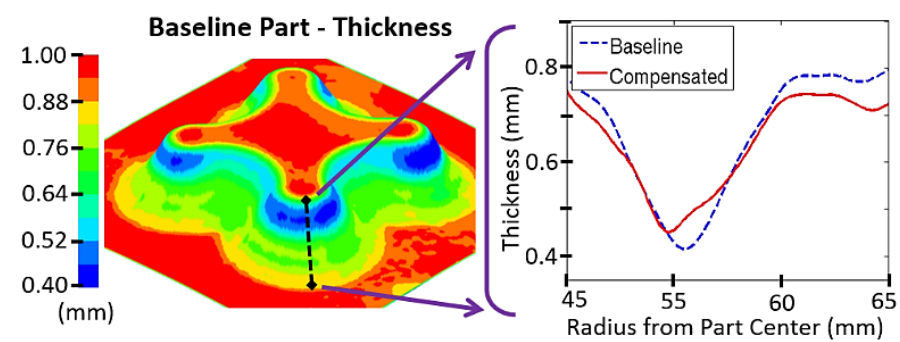

Fig. 11. Thickness distribution of Shamrock parts.

\subsection{Compensated toolpath improves formability}

To verify our hypothesis that maintaining contact delays fracture, a baseline Shamrock part (i.e., tool gap defined from the Sine law) with an increased wall angle of $65^{\circ}$ was formed, and observed to fracture (Fig. 12). Note that the compensated toolpath was able to form the entire part without fracture, thus verifying that material formability can be increased for DSIF so long as the supporting tool provides through-the-thickness pressure. It is likely that the increase in contact stress acts as a stabilizing mechanism [7], and furthermore, the compressive stress may help to partially close microcracks in the metal which effectively reduces the current state of damage.

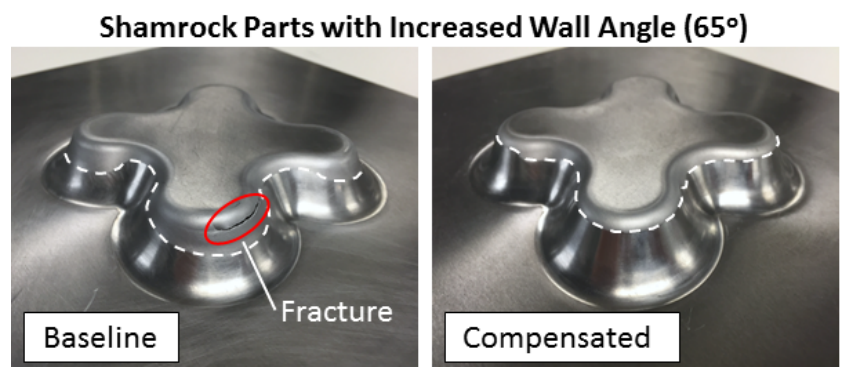

Fig. 12. Maintaining contact for DSIF increases material formability.

\section{Conclusions}

A geometry, nicknamed the 'Shamrock' part, has been developed in an effort to decouple in-plane curvature from wall angle. Using simulation and experimental studies, a new toolpath correction algorithm was proposed that incorporates forming height, in-plane curvature and tool motion direction. The algorithm effectively eliminated the loss-of-contact for the Shamrock part. By doing so, an improved distribution of sheet thickness has been observed which helps to improve material formability and prevent premature fracture. In future work, we aim to further generalize the correction algorithm as well as to investigate the local mechanics of DSIF regarding stabilization of plastic flow and the use of force control to maintain the contact.

\section{Acknowledgements}

The authors would like to acknowledge the National Science Foundation (GRFP DGE-1324585) and Department of Energy (U.S. DE-EE0005764) for their support.

\section{References}

[1] Jeswiet, J., Micari, F., Hirt, G., Bramley, A., Duflou, J., and Allwood, J., 2005, "Asymmetric Single Point Incremental Forming of Sheet Metal," CIRP Ann. Manuf. Technol., 54(2), pp. 88-114.

[2] Allwood, J. M., Music, O, Raithathna, A, and Duncan, S. R, 2009, "Closed-loop feedback control of product properties in flexible metal forming processes with mobile tools," CIRP Ann. - Manuf. Technol., 58(1), pp. 287-290.

[3] Zhang, Z., Ren, H., Xu, R., Moser, N., Smith, J., Ndip-Agbor, E., Malhotra, R., Xia, Z. C., Ehmann, K. F., and Cao, J., 2015, "A Mixed DSIF Toolpath Strategy for Improved Geometric Accuracy," ASME J. Manuf. Sci. Eng., 137, pp. 1-7.

[4] Malhotra, R., Cao, J., Ren, F., Kiridena, V., Cedric Xia, Z., and Reddy, N. V., 2011, "Improvement of Geometric Accuracy in ISF by Using a Squeezing Toolpath Strategy With Two Forming Tools," ASME J. Manuf. Sci. Eng., 133(6), p. 061019.

[5] Lu, B., Fang, Y., Xu, D. K., Chen, J., Ai, S., Long, H., Ou, H., and Cao, J., 2015 "Investigation of material deformation mechanism in DSIF," Int. J. Mach. Tools Manuf., 93, pp. 37-48.

[6] Meier, H., Magnus, C., and Smukala, V., 2011, "Impact of superimposed pressure on dieless incremental sheet metal forming with two moving tools," CIRP Ann. Manuf. Technol., 60(1), pp. 327-330.

[7] Emmens, W. C., and van den Boogaard, A. H., 2009, "An overview of stabilizing deformation mechanisms in incremental sheet forming," J. Mater. Process. Technol., 209(8), pp. 3688-3695.

[8] Moser, N., Pritchet, D., Ren, H., Ehmann, K., and Cao, J., 2016, "An Efficient and General FE Model for DSIF," ASME J. Manuf. Sci. Eng., Submitted.

[9] Martins, P. a. F., Bay, N., Skjoedt, M., and Silva, M. B., 2008, "Theory of single point incremental forming," CIRP Ann. - Manuf. Technol., 57(1), pp. 247-252. 